\title{
CFD Simulations of Open Drainage System for Offshore Applications (Caisson) using OpenFOAM
}

\author{
Zidane Moujahidi Joachim Lundberg \\ PEM-department, University of South-Eastern Norway, Norway, Joachim. I undberg@usn . no
}

\begin{abstract}
Flow assurance is of paramount importance in the oil and gas industry. In oil and gas platforms, caissons are used to discharge effluents at high volume flow rates. This latter can cause detrimental effects if they become dropped hazard objects, as they can damage subsea modules or gas pipelines. In the present work, the forces applied to a caisson due to the discharged seawater are assessed using multiphaseEulerFoam.
\end{abstract}

Keywords: caisson, OpenFOAM, VOF model, multuphaseEulerFoam

\section{Background}

Flow assurance is critical in the oil and gas industry since the flow of hydrocarbons, and other fluids should be transported successfully to ensure that the oil platform performs optimally. Interruptions of production due to any damages can cause significant financial losses.

Furthermore, in an oil platform, drainage systems are extensively present, and they can be either closed drainage systems or open drainage systems depending on the type of application. For instance, closed drainage systems, which are vented to flare, are used to collect gaseous oil and contaminated water while open drainage systems are vented to the atmosphere, and they are mainly used to dispose of seawater and noncontaminated water. Other applications of this latter include washdown water and rainwater plus process effluents and firewater (BP, 1992).

BP Group, a UK based oil and gas company, recommends taking several factors when designing or assessing open drainage systems. These factors include peak flows, hydraulic capacity, line sizing, as well as the degree of surcharge that can be tolerated (BP, 1992). In several circumstances, it is challenging to inspect a caisson, which results in inadequate assessment and little information about the state of the caisson or other equipment (BP, 2019). Nonetheless, this can be resolved by performing multiphase flow analyses to determine if there are any future potential risks.

In the context of the present study, an open drainage system i.e. a seawater caisson, is studied (Figure 1). These latter are vented tubular structures ranging over several floors designed to receive excess process streams. Also, they are used to discharge effluents continuously and intermittently with high momentum, and the flows often enter tangentially into the caisson. Noteworthy, these flows can cause detrimental effects such as exceeding the design pressure of one of the connecting supports or generating mechanical vibrations, which can, in turn, cause fatigue. Existing research on the field of multiphase flows in caissons are, to the author's knowledge, non-existing. However, some work or rotating annular flow has been investigated over the past years by Liu and Bai (2019) and Liu et al. (2020). Both studies was performed on much lower flow rates and equipment sizes than current study and the purpose of the study was to investigate the swirling flow itself rather than the forces to the surroundings.

Mohmmed et al. (2020) performed both numerical and experimental study on fluid-structure interactions from slugging multiphase flow in a horizontal pipe. The conclusion from the work was that the aeration rate affected the accuracy of the numerical models.

Stressman Engineering AS and other suppliers from the oil and gas sector are concerned about minimizing the cost and weight of the equipment, maintaining safe operation, and low maintenance. There are two cases to be addressed, in both, it is desired to quantify internal forces applied to the walls of the seawater caisson in order to determine if there are significant unbalanced forces that may cause vibrations or fatigue.

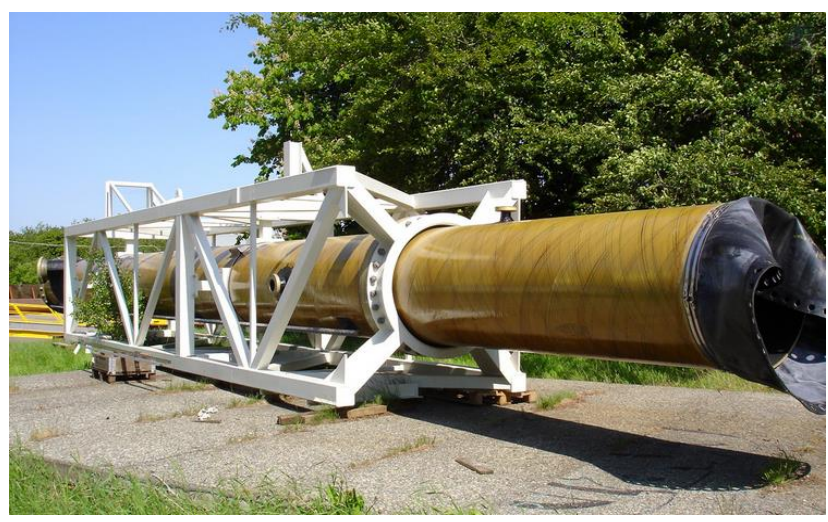

Figure 1. Seawater caisson including a support structure and membrane located in the right-hand side (White Rose, 2015). The structure is tilted horizontally in the figure. 


\section{Materials and Methods}

The first case corresponds to the maximum single continuous seawater flow during normal operations while in the second case an additional seawater flow is added, thereby flow rates in the seawater caisson are increased. Also, the cases investigated using SpaceClaim as a CAD software, OpenFOAM as a CFD simulation tool, and Paraview as a postprocessing tool. Regarding the adopted methodology, the initial solid geometry was simplified by removing the nozzles or inlets in which no flow occurred. Afterward, the fluid domain was extracted from solid geometry. Then, polygon grids were generated for each of the fluid domains. The two cases were simulated using multiphaseEulerFoam. The Eulerian approach was expected to result in more realistic flow patterns and a better depiction of phase fractions in the fluid domain.

No experimental data or comparable CFD studies was available, so the results were checked scrutinized, compared, and screened qualitatively and quantitatively.

\section{Fluid Domains and Boundary Conditions}

After simplifying the initial geometry, the fluid domain is extracted from the solid geometry of the seawater caisson using SpaceClaim software. The boundary conditions of the fluid domain are given in Table 1.

The total length of a seawater caisson is 24.34 [m], and constituted two parts. The first part, zone 1, is 21.45 [m] long, and the second part, zone 2, is 2.89 [m] long, which corresponds to the transition zone. Figure 2 illustrates the two fluid domains.

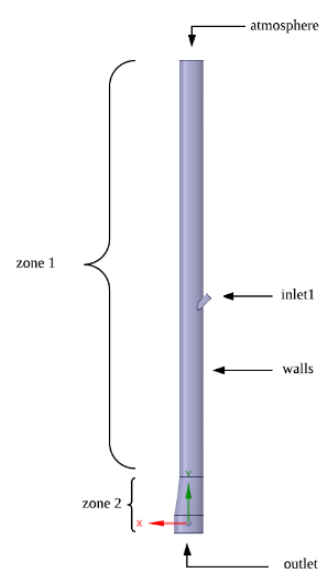

(a)

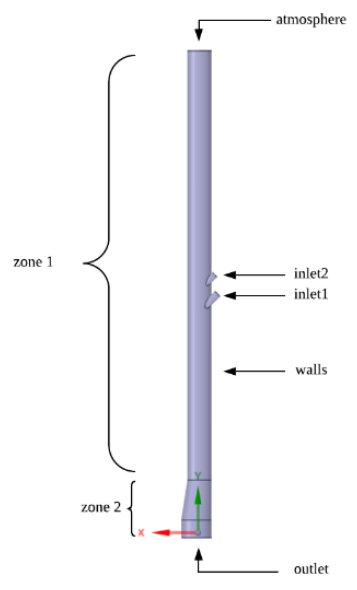

(b)
Figure 2. (a) First fluid domain; (b) second fluid domain; boundary names; and the two zones.

The inlet is inclined by $45^{\circ}$, and it has a length of 0.53 [m]. Then, it is connected to zone 1 , and it is located approximately 12.00 [m] relatively from the outlet. Noteworthy, the seawater enters the seawater caisson tangentially and, more specifically, through zone 1 . The corresponding diameters of inlet1, outlet, and the atmosphere are shown in Table 1.

Table 1. Dimensions of the patches.

\begin{tabular}{|c|c|}
\hline Boundary condition & Diameter $[\mathrm{m}]$ \\
\hline inlet 1 & 0.36 \\
\hline inlet 2 & 0.30 \\
\hline outlet & 1.50 \\
\hline atmosphere & 1.20 \\
\hline
\end{tabular}

\section{Mesh}

Initially, tetrahedral meshes were generated, and then they were converted to polyhedral meshes with a feature angle of 70 using the utility polyDualMesh 70 . Afterward, the mesh was exported as a .msh file in ASCII format.

According to Balafas (2014), one of the main advantages of polyhedral mesh over tetrahedrons is that polyhedral cells are bounded by adjacent cells, which makes the computation of gradients significantly better. Moreover, polyhedral cells are less prone to stretching compared to the other cell types making them a good candidate for preserving good mesh qualities, thereby reducing numerical diffusion and improving the accuracy of the results at a lower cell count (Balafas, 2014). Table 2 shows the mesh metrics.

Table 2. Mesh metrics of the fluid domains 1 and 2.

\begin{tabular}{|c|c|c|}
\hline Metrics & Fluid domain 1 & Fluid domain 2 \\
\hline Total number of cells & 599344 & 990071 \\
\hline Number of inflation layers & 10 & 10 \\
\hline Max. skewness & 0.590 & 0.550 \\
\hline Avg. skewness & 0.012 & 0.013 \\
\hline Max. non-orthogonality & 0.600 & 0.540 \\
\hline
\end{tabular}

Since two flows were involved in the second case study, it was expected that large gradients might occur, especially when the two flows interact at the wall of the caisson. A refined mesh was used to depict the gradients as shown in Figure 3.

Also, in the present work, the wall functions approach is used with $30<y+<200$. The employed wall functions are mainly nutkWallFunction, kqRWallFunction, and epsilonWallFunction.

Moreover, since the CFD results were verified and were found to be close to the hand calculations, no further mesh refinement was performed. In other words, the mesh independence study was not carried out. 


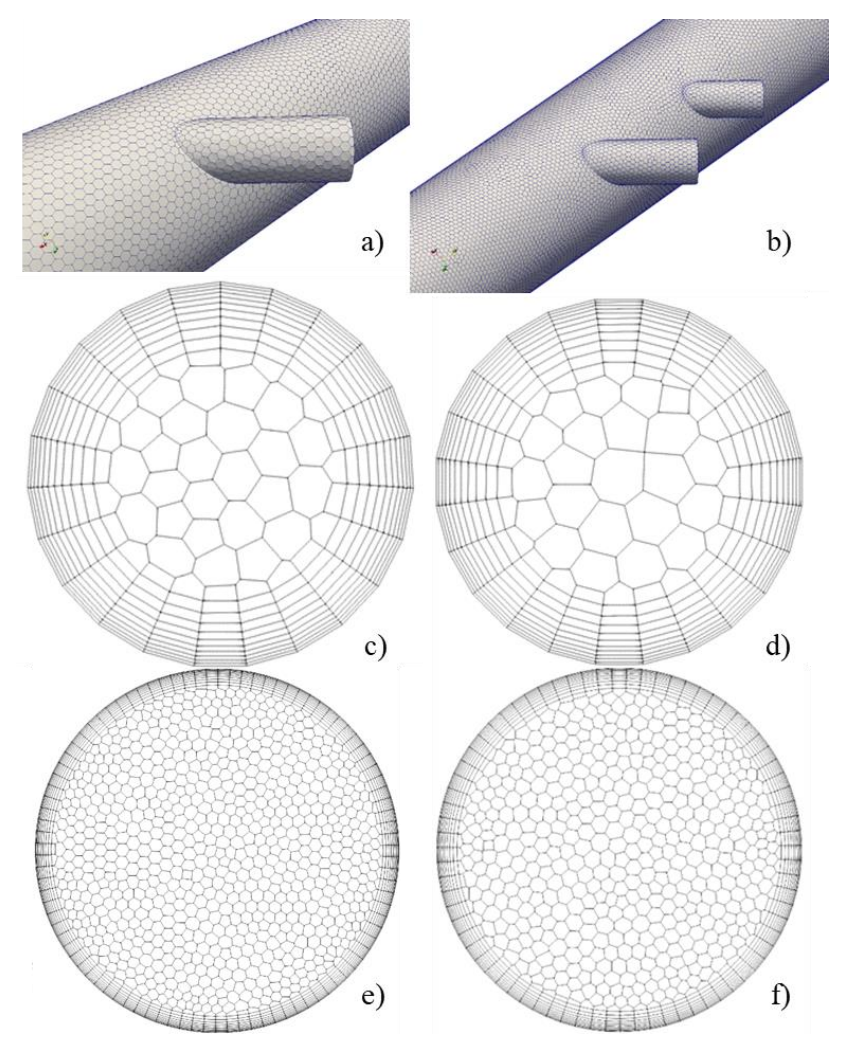

Figure 3. Meshing of the two fluid domains: (a) and (b) walls; (c) inlet1; (d) inlet2; (e) outlet; and (f) atmosphere.

\section{MultiphaseEulerFoam}

MultiphaseEulerFoam is a multiphase flow solver that can be used to solve for two or more compressible phases. It is implemented based on an Eulerian approach, and it can be coupled with the VOF interface sharpening method and turbulent models, such as RANS and LES models.

In the Eulerian approach, the phases are treated as interpenetrating continua, and the momentum equations are solved for each fluid separately; hence each fluid has its velocity field. However, the two phases have the same pressure field. Then, the momentum equations are coupled through the pressure filed, and the interface exchange momentum forces. The mass equations per phase are

$$
\frac{\partial \rho_{q} \alpha_{q}}{\partial t}+\nabla \cdot\left(\rho_{q} \alpha_{q} \boldsymbol{u}_{q}\right)=0
$$

Where

$$
\begin{array}{ll}
q & \text { seawater or air } \\
\alpha & \text { Phase fraction } \\
\mathbf{u}_{\mathbf{q}} & \text { Velocity field } \\
\rho_{\mathrm{q}} & \text { Density of } q
\end{array}
$$

Then, the momentum equations for each of the phases are

$$
\begin{aligned}
& \frac{\partial \rho_{q} \alpha_{q} \boldsymbol{u}_{\boldsymbol{q}}}{\partial t}+\nabla \cdot\left(\rho_{q} \alpha_{q} \boldsymbol{u}_{\boldsymbol{q}} \boldsymbol{u}_{\boldsymbol{q}}\right)= \\
& -\alpha_{q} \nabla p+\nabla \cdot\left(\alpha_{q} \tau_{q}\right)+\rho_{q} \alpha_{q} \boldsymbol{g}+\boldsymbol{M}_{\boldsymbol{q}}+\boldsymbol{F}_{\boldsymbol{\sigma}}
\end{aligned}
$$

Where

$$
\begin{array}{ll}
p & \text { Static pressure } \\
\tau_{q} & \text { Stress tensor } \\
M_{q} & \text { Momentum transfer } \\
F_{\sigma} & \text { Surface tension force }
\end{array}
$$

The stress tensor is expressed as

$$
\tau_{q}=\left(\mu_{q}+\mu_{t}\right)\left(\nabla \boldsymbol{u}_{\boldsymbol{q}}+\left(\nabla \boldsymbol{u}_{\boldsymbol{q}}\right)^{T}-\frac{2}{3} \nabla \cdot \boldsymbol{u}_{\boldsymbol{q}} \boldsymbol{I}\right)
$$

Where

$\begin{array}{ll}I & \text { Unit second-order tensor } \\ \mu_{q} & \text { Viscosity } \\ \mu_{t} & \text { Eddy viscosity }\end{array}$

The transport equation of the phase fraction is expressed as

$$
\frac{\partial \alpha_{q}}{\partial t}+\boldsymbol{u}_{\boldsymbol{q}} \cdot \nabla \alpha_{q}+\nabla \cdot\left(\mathbf{u}_{\boldsymbol{r}} \alpha_{q}\left(1-\alpha_{q}\right)\right)=0
$$

The third term in Equation 4 represents the compression term, and it is used instead of the interface-capturing algorithms in multiphaseEulerFoam. According to Wardle and Weller (2013), the divergence of the compression velocity $\boldsymbol{u}_{\boldsymbol{r}}$ guarantees the conservation of the volume fractions, while the term $\alpha(1-\alpha)$ restricts the effect of the interface sharpening only at the interface. Moreover, the compression term is based on the maximum velocity magnitude in the transition region, and it is expressed as

$$
\boldsymbol{u}_{\boldsymbol{r}}=\min \left(C_{\alpha}|\boldsymbol{u}|, \max (|\boldsymbol{u}|)\right) \overrightarrow{\boldsymbol{n}}
$$

And

$$
\overrightarrow{\boldsymbol{n}}=\frac{\nabla \alpha}{|\nabla \alpha|}
$$

Where

$$
\begin{array}{ll}
u_{r} & \text { Compression velocity } \\
C_{\alpha} & \text { Compression factor } \\
\overrightarrow{\boldsymbol{n}} & \text { Normal vector at the interface }
\end{array}
$$

Moreover, a continuity equation for the mixture is needed to solve for the implicit pressure equation, thus

$$
\begin{gathered}
\nabla \cdot \boldsymbol{u}=0 \\
\boldsymbol{u}=\alpha_{w} \boldsymbol{u}_{\boldsymbol{w}}+\alpha_{a} \boldsymbol{u}_{\boldsymbol{a}}
\end{gathered}
$$

\section{Simulation Setups}

In both case studies, the pressure at the inlets was set to fixedFluxPressure, and the velocities at the inlets specified using fixedValue. The velocity at inlet 1 was specified to $6.13\left[\mathrm{~m} \mathrm{~s}^{-1}\right]$ and inlet2 to $3.00\left[\mathrm{~m} \mathrm{~s}^{-1}\right]$.

For the outlet patch, prghPressure boundary condition with a static reference pressure of 101325 [Pa] was used along with the boundary condition pressureInletOutletVelocity. It switches between zeroGradient and fixedValue. zeroGadient was applied in flow directions out of the domain, while fixedValue was applied for backflow into the fluid domain.

Concerning the atmosphere patch, a common way to specify the boundary condition at this type of patch is by using totalPressure for the pressure and the 
pressureInletOutletVelocity for the velocity. totalPressure Boundary condition behaves in two ways depending on the direction of the flow. On the one hand, when there is an outflow, the pressure is set to the reference pressure, i.e., atmospheric pressure. On the other hand, when there is an inflow into the fluid domain, the pressure was computed using Equation 9 (Greenshields, 2018).

$$
p=p_{\text {ref }}-\frac{1}{2}\left|\boldsymbol{u}^{2}\right|
$$

About the initialization of the turbulence properties, i.e., $\mathrm{k}$ and $\epsilon$, at the patches were calculated and specified using Equation 10 to Equation 13. First, the Reynolds number was calculated using the inlet pipe diameter and seawater thermophysical properties.

$$
R e=\frac{\rho_{w} u_{\text {inlet }} D_{\text {pipe }}}{\mu_{w}}
$$

Secondly, according to Russo and Basse (2016), the turbulence intensity was approximated using

$$
I=0.140 R e^{-0.0790}
$$

Other equations can be used to estimate the turbulence intensity. However, it was noticed that the last equation leads to a smoother solution process. The turbulence kinetic energy and the rate of dissipation at the inlets are estimated using

$$
\begin{gathered}
k=\frac{3}{2}\left(\boldsymbol{u}_{\text {inlet }} I\right)^{2} \\
\varepsilon=C_{\mu} \frac{3}{4} \frac{k^{\frac{3}{2}}}{l}, \quad l=0.07 D_{\text {inlet }}
\end{gathered}
$$

It is important to note that the internal field was initialized using the lowest values obtained from the calculation of the turbulence properties at the inlets, in this case, inlet2. This procedure helps the solvers to compute the governing equations faster. The turbulence properties are summarized in Table 3

Table 3. The turbulence properties at the patches.

\begin{tabular}{|c|c|c|}
\hline Patches & $k\left[\mathrm{~m}^{2} \mathrm{~s}^{-2}\right]$ & $\varepsilon\left[\mathrm{m}^{2} \mathrm{~s}^{-1}\right]$ \\
\hline inlet1 & 0.140 & 0.340 \\
\hline inlet2 & 0.033 & 0.047 \\
\hline internalField & 0.033 & 0.047 \\
\hline
\end{tabular}

Furthermore, specifying the fluid properties is crucial since it predetermines the behavior of multiphase systems. It was assumed that both seawater and air are incompressible and isothermal fluids. Besides, the thermophysical properties of seawater and air are taken at $5^{\circ} \mathrm{C}$ and standard atmospheric pressure. The fluids properties are shown in Table 4

Table 4. Fluid properties.

\begin{tabular}{|l|c|c|}
\hline Fluids & $\rho\left[\mathrm{kg} \mathrm{m}^{-3}\right]$ & $v\left[\mathrm{~m}^{2} \mathrm{~s}^{-1}\right]$ \\
\hline Seawater & 1028 & $1.56 \cdot 10^{-6}$ \\
\hline Air & 1.26 & $1.37 \cdot 10^{-5}$ \\
\hline
\end{tabular}

multiphaseEulerFoam prompts the user to enter the diameter of the dispersed phase and the continuous phase. Due to the lack of data, the droplet size and bubble size are assumed to $1.00 \cdot 10^{-4}[\mathrm{~m}] 3.00 \cdot 10^{-3}[\mathrm{~m}]$, respectively.

Regarding the time stepping in the present analysis, adjustable time stepping was used and the average throughout the simulation time was found to be $0.001[s]$.

\section{Results and Discussion}

The upper halves of walls of the caisson and more specifically, above the upper inlets are not considered since the forces are expected to be insignificant. Figure 5 shows the divided walls, and the alternation between orange and white colors is for illustration purposes. The walls of the first and second fluid domains are divided into 13 slices and 14 slices, respectively. The slices are $1.00[\mathrm{~m}]$, except for the last slices located at the bottom of the caisson, which is $0.36[\mathrm{~m}]$.

Only the most descriptive slices are discussed in this paper. For more information about the forces applied to each slice are provided the work by Zidane (2020).

Additionally, in subsection 9.1 and subsection 9.2, the force plots show the net forces and the forces in the three. The black curve represents the force components along the $\mathrm{X}$-axis (axially with the inlets), the red curve represents the force components along the $\mathrm{Y}$-axis (axially with the caisson), and the blue curve represents the force components along Z-axis (perpendicular to the inlets). The green curve represents the magnitude of the forces. In the phase fraction contours, the location of the maximum net force is highlighted with a yellow dot.

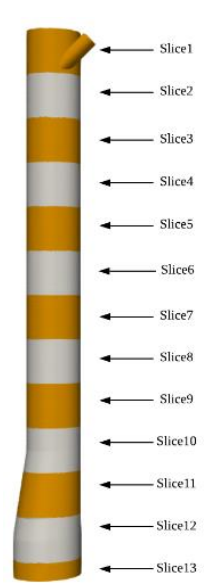

(a)

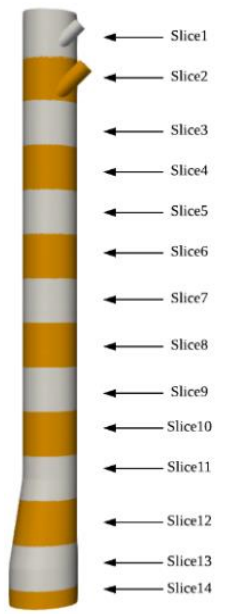

(b)
Figure 5. (a) Walls of the caisson segmented into 13 slices (first case study); and (b) walls of the caisson segmented into 14 slices (second case study).

\subsection{Evolution of peak forces - Case 1}

The seawater flow accelerates due to the $45^{\circ}$ inclination of the inlet pipe and the free fall between the exit of the pipe inlet and the wall of the large tubular structure. Besides, the seawater flow reaches $11.56\left[\mathrm{~m} \mathrm{~s}^{-1}\right]$ when 
it contacts the curved wall of the caisson. Figure 6 illustrates the net force that increases abruptly reaching a peak value of $14550[N]$ at $0.30[s]$, however, the net force decreases to $11000[N]$ and remains at this value with minor fluctuations, more specifically after $2.40[s]$. Figure 7 shows the concentration of the net force in slice 2 at 0.30 [s].

After slice2, it is observed that the forces applied to the walls of the caisson decrease, which implies that the energy of the seawater flow diffuses as the flow progresses downward. The decrease in force occurs mainly from slice4 to slice9, and it is accompanied by fluctuations that last for a certain period; however, the force fluctuations vanish thereafter.

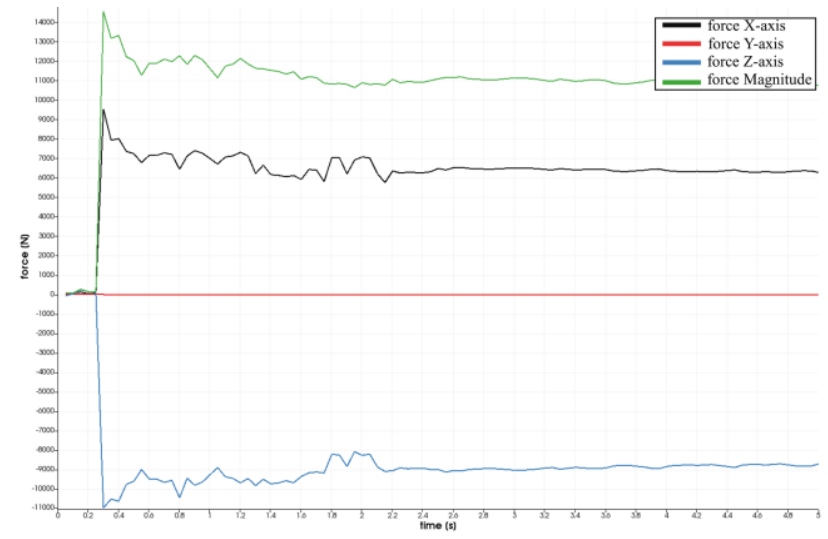

Figure 6. Slice 2 - located at $(0,-1.3,0)$.

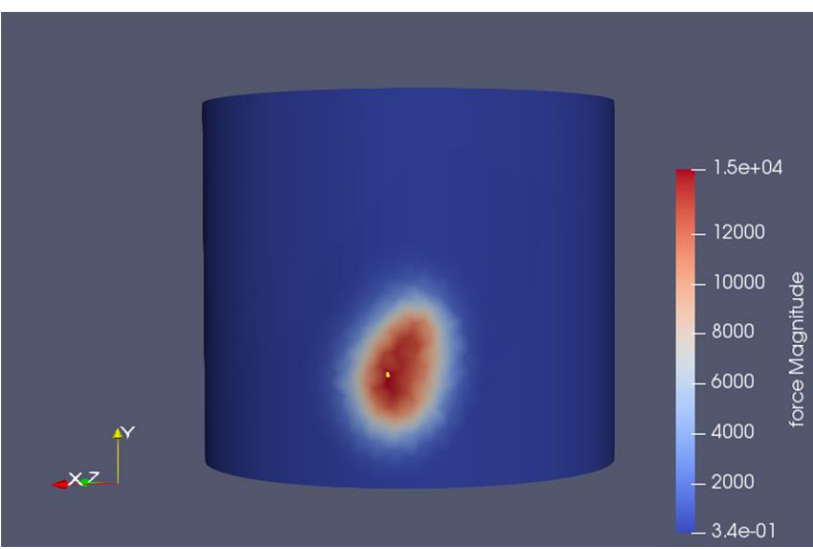

Figure 7. Force magnitude contour at 0.3 [s] in slice2located at $(0,-1.3,0)$.

Following slice11, the forces become significant again, for example in slice13, which is at the outlet of the caisson, the forces increase in a fluctuating way and become slightly stable at $13000[N]$ starting from 4.40 $[N]$, and the load imbalance is primarily due to the $\mathrm{X}$ component of the net force, as shown in Figure 8.

A critical remark is that the force imbalance in slice 13 is due to the accumulation of seawater along a line due to the swirling effect, i.e., the swirling streak. Figure 9 proves that the maximum net force coincides with the location of the swirling streak.

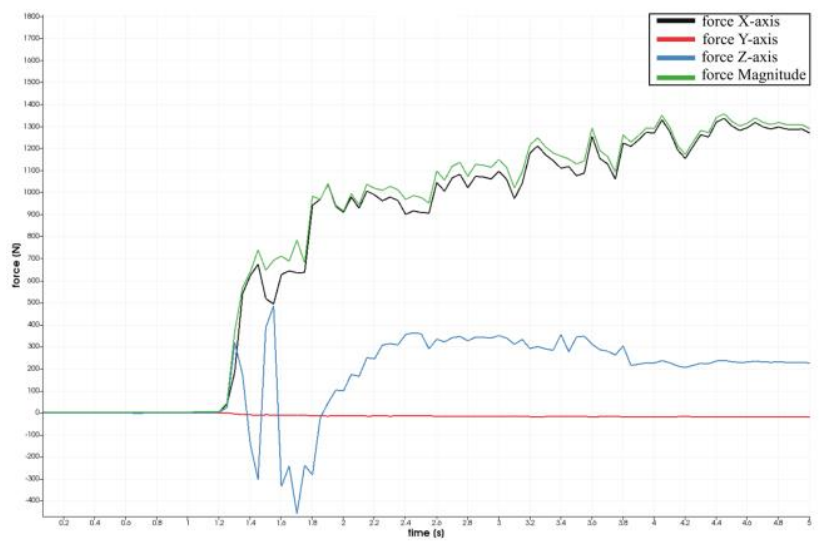

Figure 8. Slice13 - located at $(0,-12.3,0)$.

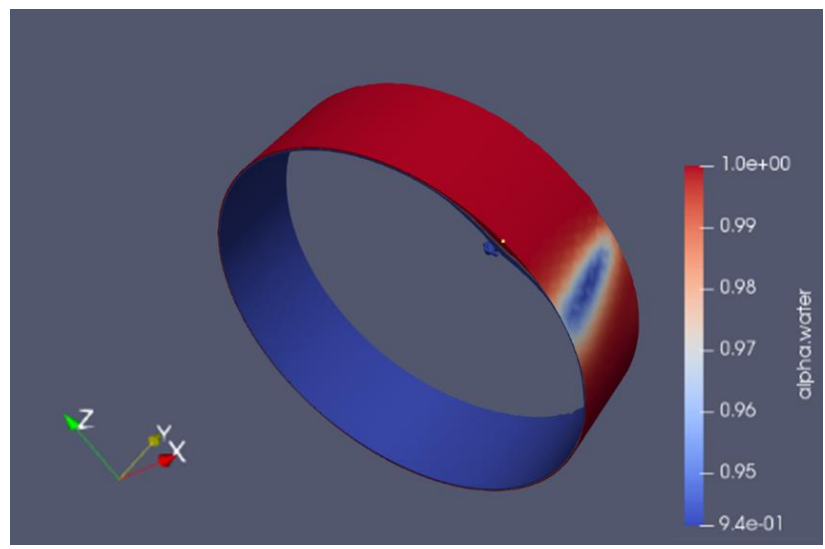

Figure 9. Seawater phase fraction contour at $5.00[\mathrm{~s}]$ in slice13 - located at $(0,-12.3,0)$.

\subsection{Evolution of peak forces - Case 2}

In slice3, the net peak force is $15980[N]$ at $0.40[s]$, which is higher than the value found in the first case study (slice2). More interestingly, the value of the net force when the flow stabilizes is $7700[N]$ which is lower than the value found in the first case of $11000[N]$. Besides, the force imbalances are due to the $\mathrm{X}$ and $\mathrm{Z}$ components of the net force. The evolution of the forces in slice 3 is shown in Figure 10.

Another interesting finding is that the momentum of the seawater at inlet1 transports the momentum of the seawater flow at inlet 2 down the caisson causing the forces to be more spread out in the slices below slice2. Also, it is noticed that the forces down the caisson display more fluctuations compared to the first case study, which is caused by the presence of the two seawater flows, i.e., instabilities, and these observations are best described using the evolution of the forces at slice5. Figure 11 shows that the maximum net force is $1550[\mathrm{~N}]$, and it occurs at $1.40[\mathrm{~s}]$ and the $\mathrm{X}$ component of the force appears to be the main contributor to the net force. Additionally, the forces exhibit large fluctuations, for example, at 2.65 [s], the fluctuation of the force along the $\mathrm{X}$-axis is about $2000[\mathrm{~N}]$. 


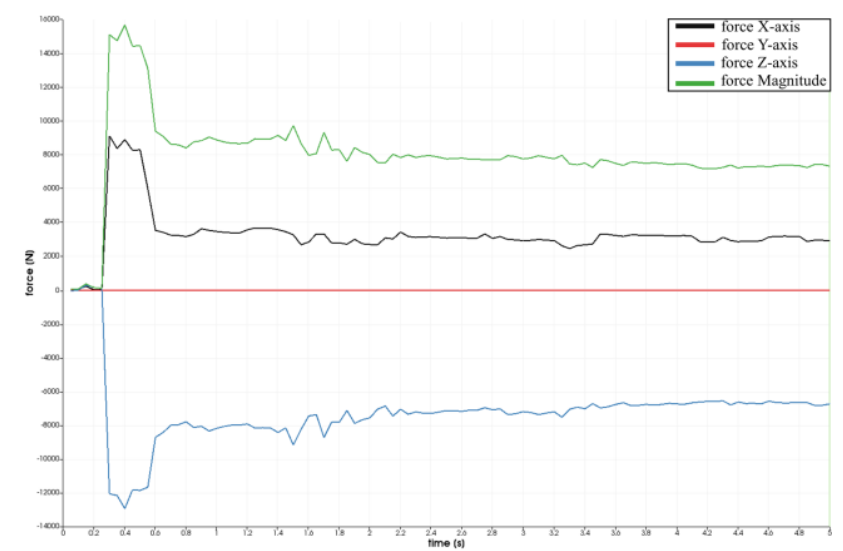

Figure 10. Slice3- located at $(0,-1.3,0)$.

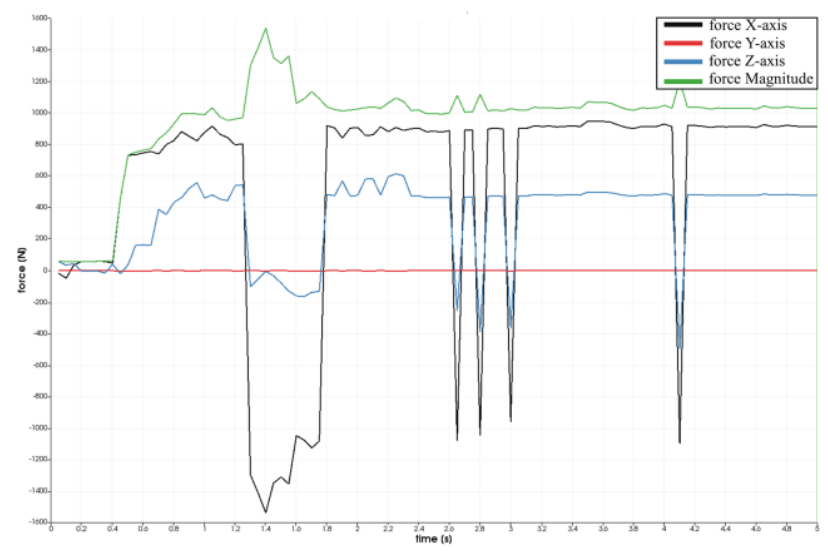

Figure 11. Slice5 - located at (0, -3.3, 0).

Interestingly, Figure 12 illustrates that the most significant force in slice 5 coincides with the leading wave of the swirling flow. Also, all the large fluctuations of forces in the $\mathrm{X}$ and $\mathrm{Z}$ axes are in the same position as swirling steak; however, when the large fluctuations disappear, and the position of the forces move to another position.

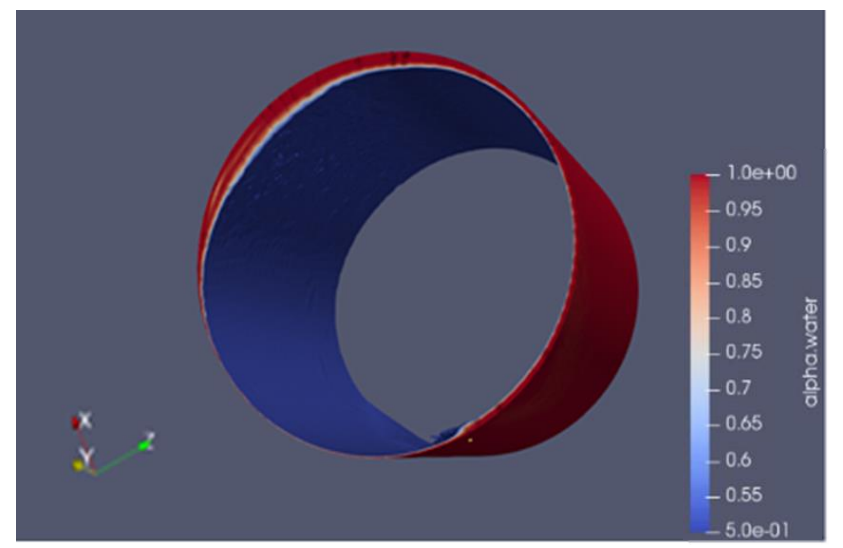

Figure 12. Seawater phase fraction contour at 1.40 [s] in slice5 - located at $(0,-3.3,0)$.

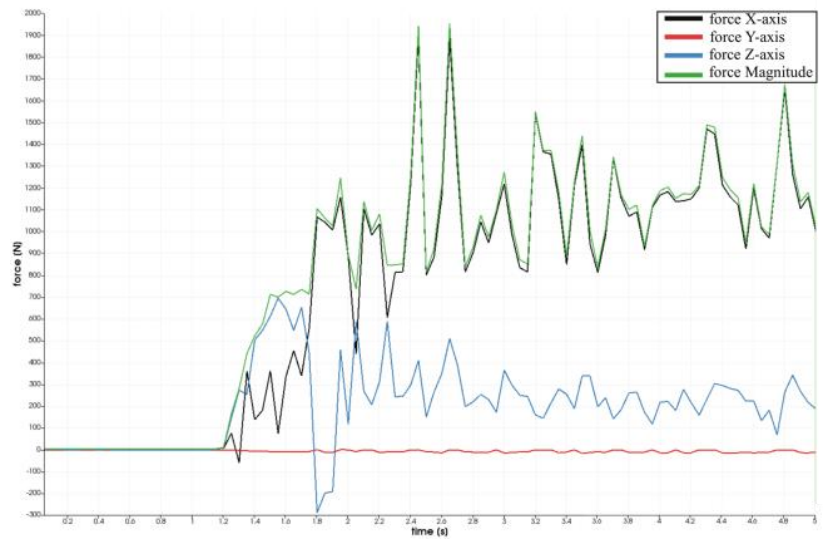

Figure 13. Slice14- located at $(0,-12.3,0)$.

It must be emphasized that the forces display similar trends as in the first case study except that the forces are higher and more fluctuating, which is caused by the presence of two seawater flows and a higher volume flow rate entering the caisson. For example, Figure 13 shows that the large fluctuations in slice14 occur primarily due to the force component in the X-direction.

Figure 14 shows that the location of the maximum force coincides with the swirling streak location, and it is the main cause of the force fluctuations in slice14.

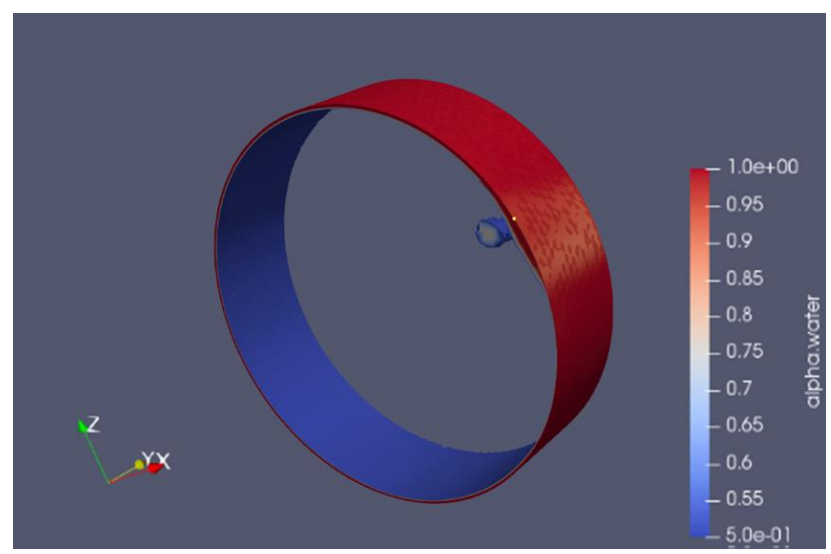

Figure 14. Seawater phase fraction contour at 5.00 [s] in slice14 - located at $(0,-12.3,0)$.

\subsection{Sanity check}

When the seawater flow is ejected from inlet1, its velocity increases up to $13.62\left[\mathrm{~m} \mathrm{~s}^{-1}\right]$ right after impacting the wall, thus the flow gains momentum. To calculate the force induced by the flow, a simple hand calculation is used

$$
\begin{gathered}
\boldsymbol{F}_{\boldsymbol{w}}=\dot{m}_{w} \boldsymbol{u}_{\boldsymbol{w}}=\rho_{w} A_{\text {inlet } 1} \boldsymbol{u}_{\boldsymbol{w}}^{2} \\
1028 \cdot\left(\pi \cdot\left(\frac{0.36}{2}\right)^{2} \cdot(13.62 \cdot \cos (45))^{2}\right) \\
=9705[N]
\end{gathered}
$$

This value is close to the $\mathrm{X}$-component of the net force in slice $2(0,-1.3,0)$, as shown in Figure 6. Besides, from the latter figure, it is observed that the force in $\mathrm{Z}$ component is slightly higher than the force in the $\mathrm{X}$ - 
component, and this can be explained by the curvature of the walls of the caisson which causes the flow to accelerate in the Z-direction more than in the $\mathrm{X}$ direction. Also, it is sensical the energy of the seawater flow diffuses as it progresses downward, except in the transition zone where it increases because of seawater accumulation and disturbances due to the change in geometry.

Additionally, from a qualitative perspective, the flows in the caisson behavior appear to be physical, i.e., vertical downward annular flows and the presence of swirling streak as found by Liu et al. (2020).

\section{Conclusions}

From the CFD simulation results of the two cases, the following conclusions are drawn:

- The magnitude of the forces and their distribution in the caisson are strongly dependent on the volume flow rates and the number of the inlets.

- The main forces are acting in the radial direction whereas the forces in the axial direction are nonexistent in all the parts of the caisson and throughout all the simulation times.

- In the first case study, the largest forces are located where the seawater interacts with the walls of the caisson for the first time, namely, in slice 2 and slice 3 and slice13. Then, as the flow of seawater progresses, the forces decrease in magnitude, implying that the energy of the seawater is diffused. However, when the seawater reaches the transition zone, the forces further increase and display more fluctuations as a result of the increased momentum of the seawater flow, flow accumulation, and flow disturbances.

- In the second case, the forces have similar trends as in the first case, but they are larger and more fluctuating, and they are more distributed throughout the lower section of the caisson. This happens because the seawater flow from inlet 2 interferes with the seawater flow from inlet1, causing more instabilities in the seawater flow. Also, this interference causes the propagation of seawater flow momentum in the downward direction, hence the different force distributions between the first and second cases

- In both cases, these forces exhibit fluctuations; however, they vanish afterward.

- The mass flow rates through the caisson increase exponentially and reach pseudo-steady states after the flow establishes.

- It is noticed that air is sucked from the atmospheric boundary due to the downward momentum of the seawater flow.

- The first and third cases show good agreement with the hand calculations.

- All the simulations show reasonable flow patterns which are in good agreement with flow phenomena mentioned in the literature review.
- Since large, imbalanced, and fluctuating forces are found in the case studies, it is recommended to use the CFD results in present work to perform FEA analyses to quantify the resulting vibrations and fatigues.

\section{References}

G. Balafas. Polyhedral Mesh Generation For CFD - Analysis Of Complex Structures. Master's thesis, Technical University of Munich, Munich, Germany, 2014.

BP. Azeri Central East Project: Environmental and Social Impact Assessment, Non-Technical Summary, BP, 2019.

BP. Guide to the Selection, Arrangement and Specification of Offshore Platform Drainage. Engineering Practices Group, BP International Limited, Middlesex, United Kingdom, 1992.

C. Greenshields. Openfoam V6 User Guide: 5.2 Boundaries. CFD Direct, Retrieved from https://cfd.direct/openfoam/ user-guide/v6-boundaries. July $10^{\text {th }} 2018$.

L. Liu and B. Bai. A mechanistic model for the prediction of swirling annular flow pattern transition. Chemical Engineering Science, 199:405-416, 2019. doi: 10.1016/j.ces.2019.01.039.

W. Liu, X. Lv and B. Bai. Axial development of air-water annular flow with swirl in a vertical pipe. International Journal of Multiphase Flow, 124, 2020. doi: 10.1016/j.ijmultiphaseflow.2019.103165.

A. O. Mohmmed, H. H. Al-Kayiem. A. B. Osman and O. Sabir. One-way coupled fluid-structure interaction of gasliquid slug flow in a horizontal pipe: Experiments and simulations. Journal of Fluids and Structures, 97, 2020. doi: 10.1016/j.jfluidstructs.2020.103083.

F. Russo and N. T. Basse. Scaling of turbulence intensity for low-speed flow in smooth pipes. Flow Measurement and Instrumentation, 52:101-114, 2016. doi: 10.1016/ j.flowmeasinst.2016.09.012.

K. E. Wardle and H. G. Weller. Hybrid Multiphase CFD Solver for Coupled Dispersed/Segregated Flows in LiquidLiquid Extraction. International Journal of Chemical Engineering, 2013:1-13, 2013. doi: 10.1155/2013/128936.

White Rose. K30 Storage Process Description, Technical: Storage. UK CCS Commercialisation Programme, United Kingdom, December, 2015.

M. Zidane. CFD Simulations of Open Drainage System for Offshore Applications (Caisson) Using Openfoam. Master's thesis, University of South-Eastern Norway, Porsgrunn, Norway, 2020. 